\title{
Innovationen in der Senologie
}

\section{Moderne Senologie aus Sicht der plastischen Chirurgie}

\author{
Mario Marx
}

Dank der Etablierung von interdisziplinären zertifizierten Brustzentren wurden in Deutschland 2010 mehr als 80\% der an Brustkrebs erkrankten Frauen gemäß der S3-Leitlinie für Diagnostik und Therapie des Mammakarzinoms beraten und behandelt.

Die in der S3-Leitlinie geforderte Interdisziplinarität zur Gewährleistung einer umfassenden Beratung und Behandlung der sich uns anvertrauenden Patientinnen wird zunehmend mit Leben gefüllt. Enge fachliche Vernetzungen zwischen Kollegen der Gynäkologie und der plastischen Chirurgie führen zu einer besseren Abstimmung von onkologischem und rekonstruktiven Konzept.

Die S3-Leitlinie fordert von den onkologischen Operateuren eine nachgewiesene Expertise und Mindestzahlen. Um dem hohen Anspruch evidenzbasierter Therapie auch von unserer Seite gerecht zu werden, hat die Deutsche Gesellschaft der Plastischen, Rekonstruktiven und Ästhetischen Chirurgen (DGPRÄC) beschlossen, sich den selben Anforderungen zu stellen.

Unter der Leitung von Herrn Dr. Uwe v. Fritschen (Berlin) wurde in den vergangenen 3 Jahren ein Curriculum erarbeitet, dass neben einer umfassenden operativen Ausbildung auch eine jährliche Mindestzahl von rekonstruktiven Brusteingriffen vorsieht. Zusätzlich erfolgt verbindlich die prospektive, externe Erfassung und Beurteilung der Ergebnisqualität. Rekonstruktiven Zentren, die die umfangreichen Anforderungen erfüllen, vergibt die Fachgesellschaft ein Qualitätssiegel.

Hiermit wird es möglich, die erbrachte plastisch-rekonstruktive Leistung unter genormten Standards zu erfassen und mit anderen Kliniken zu vergleichen. Der Vorteil ist eindeutig bei den Patientinnen, welche ebenso wie die geprüfte Qualität der zertifizierten Brustzentren die Qualität und Quantität der plastischen Chirurgen einsehen können. Die Pilotphase ist abgeschlossen und die Zertifizierung der plastisch-rekonstruktiven Zentren der DGPÄC wird dieses Jahr beginnen.

Unbestritten ist die Anwendung der verschiedenen PerforatorLappen wie DIEP ( Abb. 1), S-GAP, I-GAP, SIA-Flap, FCI -Lappen u.a. mittlerweile in die klinische Routine überführt. Mehr als 1000 freie Lappenplastiken wurden in 2010 zur Brustrekonstruktion in der Bundesrepublik (Register der DGPRÄC) durchgeführt.

Die neue Klassifikation onkologischer, rekonstruktiver und onkoplastischer Operationsverfahren beim Mammakarzinom nach Komplexitätsgraden von J.Hoffmann und D.Wallwiener (Tübingen) bietet eine strukturierte Grundlage zur Implementierung der zu assoziierenden rekonstruktiven Verfahren. Auf dieser Basis kann die indikationsbezogene und sichere operative Umsetzung dieser Techniken erfolgen. Sie erfordern neben einer gründlichen mikrochirurgischen Ausbildung mit Facharztstandard eine jahrelange, nicht endende kritische und ehrfürchtige Weiterentwicklung der Operateure.

Die Indikationen für diese Techniken sind sehr vielfältig. Nach Brust-Amputation und Wunsch unserer Patientinnen ist die ein-

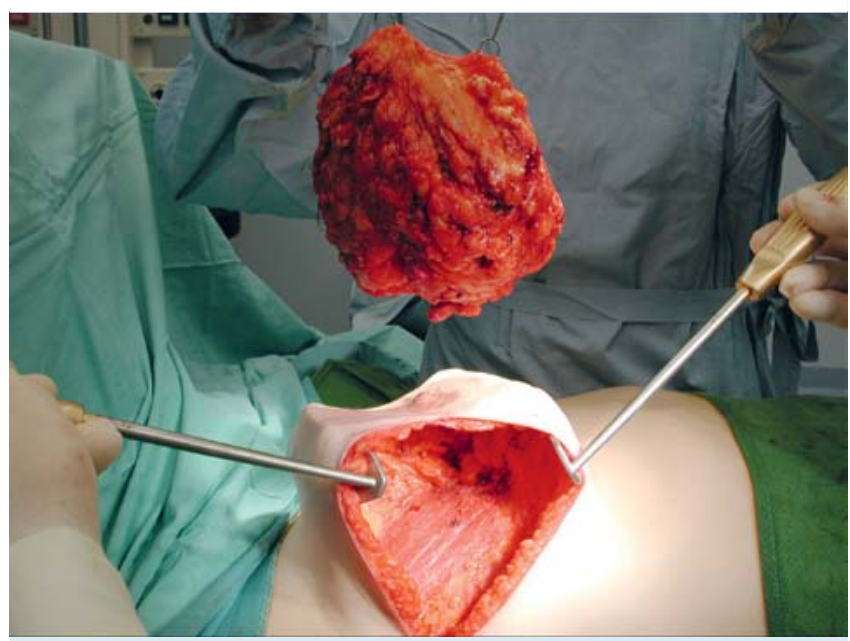

Abb. 1 Skin-conserving-Mastektomie - Drüsenkörperextirpation ventral der Fascia Scarpa. 862 g-Brustdrüse mit $8 \mathrm{~cm}$ großem DCIS.

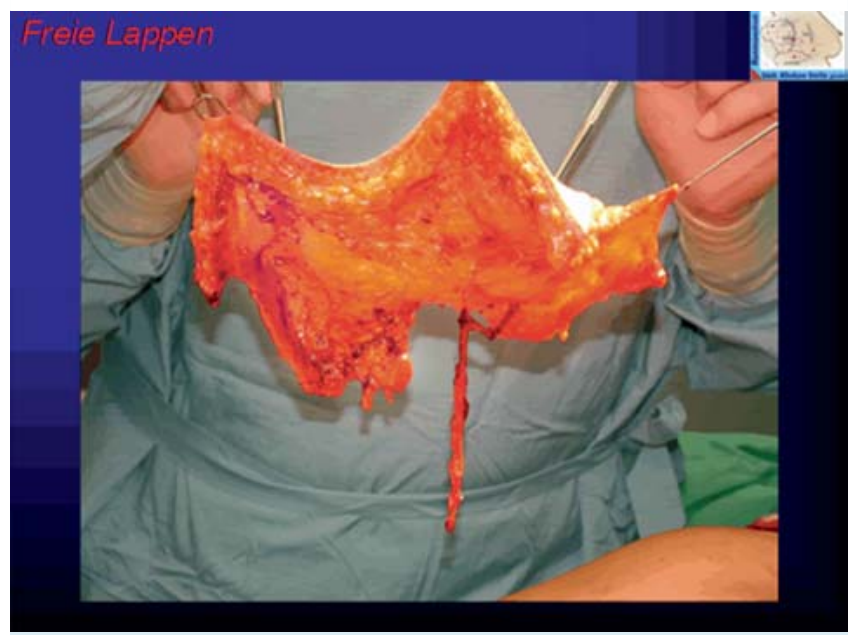

Abb. 2 Bipedikulärer DIEP-Flap.

oder beidseitige sekundäre Wiederherstellung mittels Eigengewebe inzwischen Standard.

Bei der Skin-conserving-Mastektomie wird die Brustdrüse vollständig ventral der Scarpa'schen Faszie entfernt. Diese Technik wird auch von den gynäkologischen Operateuren zunehmend eingesetzt und erfordert für ein dauerhaft befriedigendes Resultat in aller Regel eine Rekonstruktion mit Eigengewebe (D Abb. 2).

Ca. 4-6\% der Frauen sind BRCA1/2-Mutationsträgerinnen. Hier ist eine intensive Zusammenarbeit mit dem aktuell erweiterten Netzwerk für erblich bedingte Brusterkrankungen in der Bundesrepublik Deutschland erforderlich. Derzeit werden den Pa- 
tientinnen unterschiedliche operative Konzepte angeboten. Hier kann prospektive wissenschaftliche Kooperation beim Angebot plastisch-rekonstruktiver Verfahren zum größten Vorteil dieser Patientengruppe werden.

Zahlreiche Herausforderungen können wir gemeinsam bewältigen. Dazu gehört exemplarische das DCIS, bei dem Über- und Untertherapie vermieden werden müssen. Die Vielfalt der rekonstruktiven Optionen mit Silikon-Implantaten und Expandern in Kombination mit weiteren heterologen Materialen erweitert das Angebot an Möglichkeiten zur Brustrekonstruktion. Oder stellen wir uns der Frage, ob neue Technologien der Eigenfettimplantation unter Berücksichtigung der speziellen onkologischen Herausforderungen an der Brust in Diagnostik und Therapie in den kommenden Jahren ihre Implementierung erfahren werden? Die Wiederherstellung des Mammillen-Areola-Komplexes bietet ebenfalls eine große Vielzahl an technischen Möglichkeiten und ein breites Feld für Innovationen.

Nur wenn es uns gelingt, eine partizipierte Partnerschaft anzubieten, können wir die Innovationen unserer Fachgebiete vollumfänglich unseren Patientinnen und Patienten anbieten.

Das Angebot, sich Zweitmeinungen einzuholen oder die operative Strategie im Vorfeld abzustimmen, ist keine Schwäche des Empfehlenden und wird im Gegenteil von den Patientinnen als besonders gewissenhaft wahrgenommen.
Ich wünsche unserer 31. Jahrestagung einen kreativen und erfolgreichen interdisziplinären Wissensaustausch zum Wohle unserer Patientinnen und Patienten.

\section{Literatur}

1 Hoffmann J et al. Classifying breast cancer surgery: a novel, complexity-based system for oncological, oncoplastic and reconstructive procedures, and proof of principle by analysis of 1225 operations in 1166 patients. BMC Cancer 2009; 9: 108

\section{Bibliografie}

DOI http://dx.doi.org/10.1055/s-0031-1271501

Senologie 2011; 8: 79-80

(c) Georg Thieme Verlag KG Stuttgart · New York · ISSN 1611-6453

\section{Korrespondenzadresse}

Dr. med. Mario Marx

Städtisches Klinikum Görlitz gGmbH

Ärztlicher Direktor der

Klinik für Plastische, rekonstruktive und Brustchirurgie

Direktor zertifiziertes Mammazentrum

Girbigsdorfer Str. 1-3

02828 Görlitz

marx.mario@klinikum-goerlitz.de 